\title{
Морфофункциональное состояние нижнечелюстных костей лабо- раторных белых крыс при 1- и 3-месячном воздействии бис- фосфонатов в дозах, вызывающих остеонекротические изменения
}

\author{
Семен Мостовой ${ }^{1}$ Василий Пикалюк $^{2}$ \\ ${ }^{1}$ Крымская медицинская академия имени С. И. Георгиевского, Симферополь, Украина \\ ${ }^{2}$ Восточно европейский национальный университет имени Леси Украинки, Луцк, Украина \\ Адрес для переписки: semen-34@ yandex.ru
}

Отримано: 02.05.19; прийнято до друку: 03.06.19; опубліковано: 28.06.19

\begin{abstract}
Резюме. Известно, что препараты группы бисфосфонатов могут вызывать тяжелые осложнения остеонекрозы челюстей. Однако воздействия этих соединений в дозах, вызывающих остеонекротические поражения, на рост и формообразование нижнечелюстных костей остаются малоизученными. Целью работы явилось рассмотрение морфофункционального состояния (составляющих размера и формы) нижнечелюстных костей на фоне приёма бисфосфонатных соединений в дозах, вызывающих остеонекротические поражения. Исследование проводили на 60 белых беспородных крысах, которым вводили исследуемые соединения. Объектом исследования явились нижнечелюстные кости. Производилось сканирование исследуемых объектов, их оцифровка и определение 22 ландмарок. Анализ составляющей формы (геометрическая морфометрия) производился в программах TPSDig и MorphoJ. Цифровые данные, характеризующие линейные размеры, анализировались в программе Statistica 10.0 с использованием одно-, двух- и многофакторного дисперсионных анализов (ANOVA, MANOVA), а также апостериорного критерия Ньюмана-Кеулса. Выявлено, что в ранние сроки воздействия исследуемые бисфосфонатные соединения обладают выраженным остеостимулирующим действием, несмотря на наличие «реакции острой фазы», возникающей в первые две недели использования. В поздние сроки исследования вещества вызывают торможение роста и нарушение процессов формообразования, что обусловлено фармакологическими свойствами исследуемых веществ, блокированием костной резорбции и широким спектром возможных токсических реакций со стороны мочевыделительной, пищеварительной и сердечно-сосудистой систем.
\end{abstract}

Ключевые слова: нижняя челюсть, бисфосфонаты, остеонекрозы, геометрическая морфометрия.

\section{Morpho-Functional Condition of the Mandibular Bones of Labora- tory White Rats at 1- and 3-Months of Exposure to Bisphosphonates in Doses that Cause Osteonecrotic Changes.}

\author{
Semen Mostovoy ${ }^{1}$, Vasyl Pykaliuk ${ }^{2}$ \\ ${ }^{1}$ Medical Academy named after S. I. Georgievsky, Simferopol, Ukraine \\ ${ }^{2}$ Lesya Ukrainka Eastern European National University, Lutsk, Ukraine \\ Correspondence: semen-34@yandex.ru
}

\begin{abstract}
It is known that medications of the bisphosphonate group can cause severe complications such as osteonecrosis of the jaws. However, the effects of these compounds in doses that cause osteonecrotic lesions on the growth and shaping of the mandibular bones remain unstudied.The aim of the investigation was to study the morphofunctional condition (size and shape) of the mandibular bones on the background of taking bisphosphonate compounds in doses that cause osteonecrotic lesions. The research was performed on 60 white
\end{abstract}


outbred rats that were exposed to the studied remedies. The objects of the study were mandibular bones. They were scanned, digitized and 22 landmarks were determined. The analysis of the form component (geometric morphometry) was carried out with the programs TPSDig and MorphoJ. Digital data characterizing linear dimensions were analyzed due to the program Statistica 10,0 using one-way analysis, two-way analysis and multivariate analysis of variance (ANOVA, MANOVA) and Newman-Keuls post-hoc test. It was revealed that in the early stages of exposure, the studied bisphosphonate compounds have a significant effect on osteostimulation, despite the «acute phase reaction» that takes place during the first two weeks of use. At the later dates of the study, the substances cause growth inhibition and disorder of shaping processes because of the pharmacological properties of the studied substances, blocking bone resorption and a wide range of possible toxic reactions of the urinary, digestive, and cardiovascular systems.

Key words: mandible, bisphosphonates, osteonecrosis, geometric morphometry.

\section{Введение}

Остеотропные свойства соединений фосфора известны ещё с XIX в. Часть из них (бисфосфонаты, аминобисфосфонаты) используется для лечения нарушений костного метаболизма. Способность предупреждать значительную потерю костной массы позволяет их включать их комплексную терапию в лечении системного остеопороза. Кроме того, эти вещества применяются в онкологии для терапии костных метастазов, сочетающихся с синдромом гиперкальциемии. Однако известно, что эти препараты могут вызывать тяжелые осложнения остеонекрозы челюстей $[1,2,3]$. Подобные процессы исследователи наблюдают у лиц, употребляющих метамфетамин кустарного производства, при изготовлении, которого применяется красный фосфор. В наших более ранних исследованиях приведе-ны результаты, доказывающие бисфосфонат-ную природу примесей, образующихся при синтезе метамфетамина кустарным способом, которые являются причиной развития остеонек-ротических процессов в челюстных костях [4].

В эксперименте на белых крысах детально изучены процессы роста и формообразования костной ткани челюстей при воздействии терапевтических доз аминобисфосфонатов, при которых не отмечается возникновение остеонекротических поражений. Нами рассмотрены гистоморфоструктурные изменения, происходящие в трубчатых и нижнечелюстных костях при дозировках аминобисфосфонатов, которые приводят к явлениям асептических остеонекротических поражений [5]. Однако морфофункциональное состояние нижнечелюстных костей на фоне приёма этих соединений в дозах, вызывающих остеонекротические поражения, остаются открытыми.

Цель работы - изучение морфофункционального состояния (составляющих размера и формы) нижнечелюстных костей на фоне приёма бисфосфонатных соединений в дозах, вызывающих остеонекротические поражения.

\section{Материалы и методы}

Экспериментальные исследования проводились на 60 белых беспородных крысах-самцах с массой тела 150-200 граммов. Животные разделены на три равные группы. Первую и вторую составили 20 трехмесячных животных, которым 1 раз в супки в течение 1-го и 3-х месяцев проводили внугрибрюшинное введение 1,0 мл физиологического раствора (1 и 2 серии опыта).

Третья и четвертая группы - 20 трехмесячных животных, которым 1 раз в сутки в дозе 63 мг/кг в течение 1- и 3-х месяцев внутрибрюшинно вводили препарат аминобисфосфонатной групाы «Памиред» (памидроновая кислота).

Пятую и 6 группы составили 20 животных, которым на протяжении 1- и 3-го месяцев в дозе 63 мг/кг внутрижелудочно вводили модельную примесь, образующуюся при кустарном изготовлении метаамфитамина. Дозу подбирали с учетом пероральной дозы, используемой наркозависимыми лицами (до 700 мг в сутки), с учётом видовых различий в скорости метаболизма в организме человека и лабораторной крысы [6,7].

Животных выводили из эксперимента под кетаминовым наркозом из расчета 4,4 мг/кг на 30- и 90-е сугки в соответствии с международными правилами проведения работ с экспериментальными животными путем декапитации [8]. Материалом для исследования служили скелетированные нижние челюсти крыс. Их подвергали сканированию с помощью сканера Epson 3490в разрешении 300 dpi.

Для облегчения анализа все полученные изображения правых нижних челюстей были отображены по горизонтали в программе «Adobe Photoshop CS5». Для характеристики формы и размера нижней челюсти использовали 22 меток-ландмарок (рис. 2), которые размещали на изображениях и оцифровывали при помощи экранного дигитайзера tpsDig2 [9]. Для анализа формы исходные координаты экземпляров нормировали относительно эталонной конфигурации с помощью метода прокрустова наложения и проводили 
Морфофункииональное состояние нижнечелюстных костей лабораторных белых крыс при 1- и 3-месячном воздействии бисфосфонатов в дозах, вызываютих остеонекротические изменения

статистическую обработку в программе MorphoJ c использованием канонического анализа, позволяющего построить такие линейные комбинации в двух заданных группах признаков, что обычный коэффициент парной корреляции между этими комбинациями достигает наибольшего значения [10]. Для определения размерной составляющей в программе TPSDig с помощью инструмента линейки рассчитывали координаты, характеризующие положение ландмарок, которые преобразовывали в линейные размеры. С целью редукции данных использован факторный анализ. Для оценки функционального потенциала конфигураций нижней челюсти использовали четыре промера: А - сочленовнорезцовый, В - сочленовно-молярный, С темпорально-сочленовный, D - сочленовномассетерный (см. рис. 1). По этим промерам вычисляли четыре индекса: темпоральнорезцовый $-T I=C / A$; темпорально-молярный $T I=C / B ;$ массетерно-резцовый $-M I=D / A$; массетерно-молярный $-M M=D / B[11,12]$.

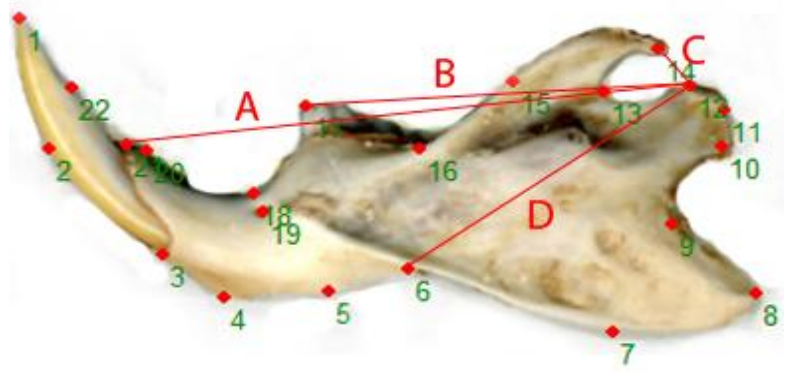

Рис. 1. Схема расположения ландмарок нижнечелюстной кости, используемых для изучения составляющих размера, формы и промеров, необходимых для вычисления морфофункииональных индексов:

$A-$ резцовый, $B$-молярный,

$C$ - темпоральный, $D$-массетерный.

В работе [13] для оценки прочности нижнечелюстных костей мы применили индекс прочности (Index of Robusticity), предложенный Розенфельдом, который использовал с этой целью индекс Пондера (Ponderal Index): $\mathrm{P}_{\mathrm{I}}=$ $\mathrm{L} / 3 \sqrt{\mathrm{m}}$, (L - длина и $\mathrm{m}$ - масса исследуемого объекта). Однако, по сути, этот индекс адекватно описывает линейные одномерные объекты, которыми являются, например, трубчатые кости. Учитывая сложность конфигурации челюсти, более естественно ее интерпретировать как двумерный объект. На случай двумерных объектов приведенную формулу можно обобщить: $\mathrm{P}_{\mathrm{S}}=\mathrm{S} /\left({ }^{3} \sqrt{\mathrm{m}}\right)^{2}$.
Площадь определяли в среде программы для анализа изображений Image Pro Plus 7.0. Статистическую проверку полученных результатов проводили при помощи одно-, двух- и многофакторного дисперсионных анализов (ANOVA, MANOVA) при уровне значимости $\mathrm{p} \leq 0,05$. Анализ соответствия вида распределения признаков закона нормального распределения проводили с использованием критерия Шапиро-Уилка $(p>0,05)$. Так как распределения значений признаков в группах соответствовали нормальному распределению, значимость различий оценивали, используя параметрические критерии. Однородность дисперсий проверяли при помощи критерия Левена. Сравнения между сериями осуществляли, используя апостериорный критерий Ньюмана-Кеулса, при уровне значимости $\mathrm{p}>0,05$. Цифровые данные положены в основу таблиц и графиков. В работе использовались пакеты прикладных программ MorphoJ TPS 2.0. и Statistica 10.0.

\section{Результаты исследования}

Предварительный анализ показал, что различия, связанные с повторной расстановкой меток и повторным получением изображений, оказались статистически недостоверны, что позволяет ими пренебречь. Тем не менее, в дальнейшем использованы усредненные индивидуальные координаты меток по трём повторам. Канонический анализ оцифрованных изображений нижнечелюстных костей исследуемых выборок построен на основе координат, полученных методом прокрустова наложения, выявил значительные межвыборковые различия (табл. 1; рис. 2.) Полученные две канонические переменные CV1 - 81,676 \% и CV2 - 18,324 \% объясняют все изменение формы исследуемых челюстных костей. Графически это представлено диаграммой рассеивания объектов, в плоскости двух канонических переменных в виде трех неперекрывающихся чётко разграниченных облаков множеств (рис. 3). Облако, имеющее высокий морфологический градиент по второй канонической оси, связано с контрольной группой животных, объединяя (1 и 2) группы. Второе облако имеет самый низкий морфологический градиент по первой и второй каноническим осям и связано с группой животных, получавших памидроновую кислоту, объединяя третью и четвёртую группы. Третье облако занимает промежуточное положение между множествами, характе- 
ризующими первую и вторую исследуемые группы по отношению ко второй канонической оси, и самый высокий градиент - по отношению к первой канонической оси. Оно связано с четвёртой и пятой сериями животных, получавших модельную примесь.

По первой канонической переменной отмечается смещение по 1, 2, 3, 4, 9, 10, 11, 13, $16,18,19,20,22$ ландмаркам. По второй канонической переменной - смещение по 1, 2, 4, 5,
$8,9,10,11,13,16,18,19,20,21,22$ ландмаркам (табл 1). Облако контрольной группы можно рассматривать как показатель выражен-ности рельефа нижнечелюстной кости и пропорциональности её анатомиических образований, а облака исследуемых групп - как показатели степени размытости рельефа и диспропорции в развитии анатомических отделов нижнечелюстных костей, возникшие под действием исследуемых препаратов (рис. 2).

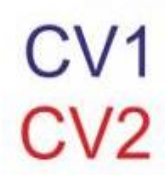

Прокрустовы координаты

Координаты по первой канонической переменной

Координаты по второй канонической перементой

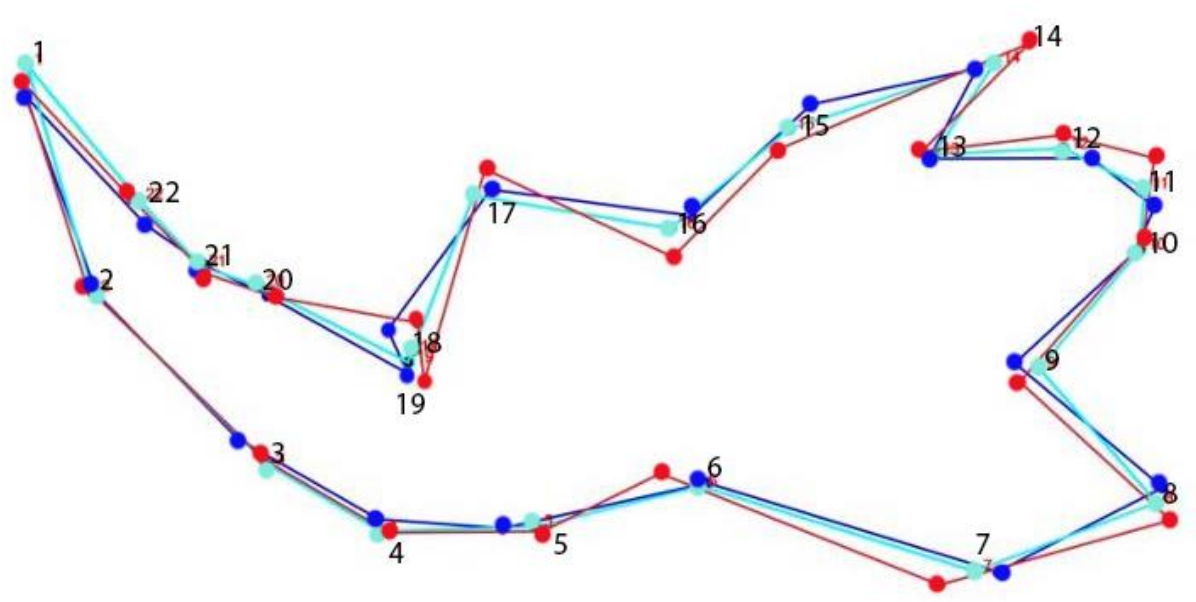

Рис. 2. Расположение каркасньх моделей нижнечелюстной кости относительно контура, построенного на основе Прокрустовых координат

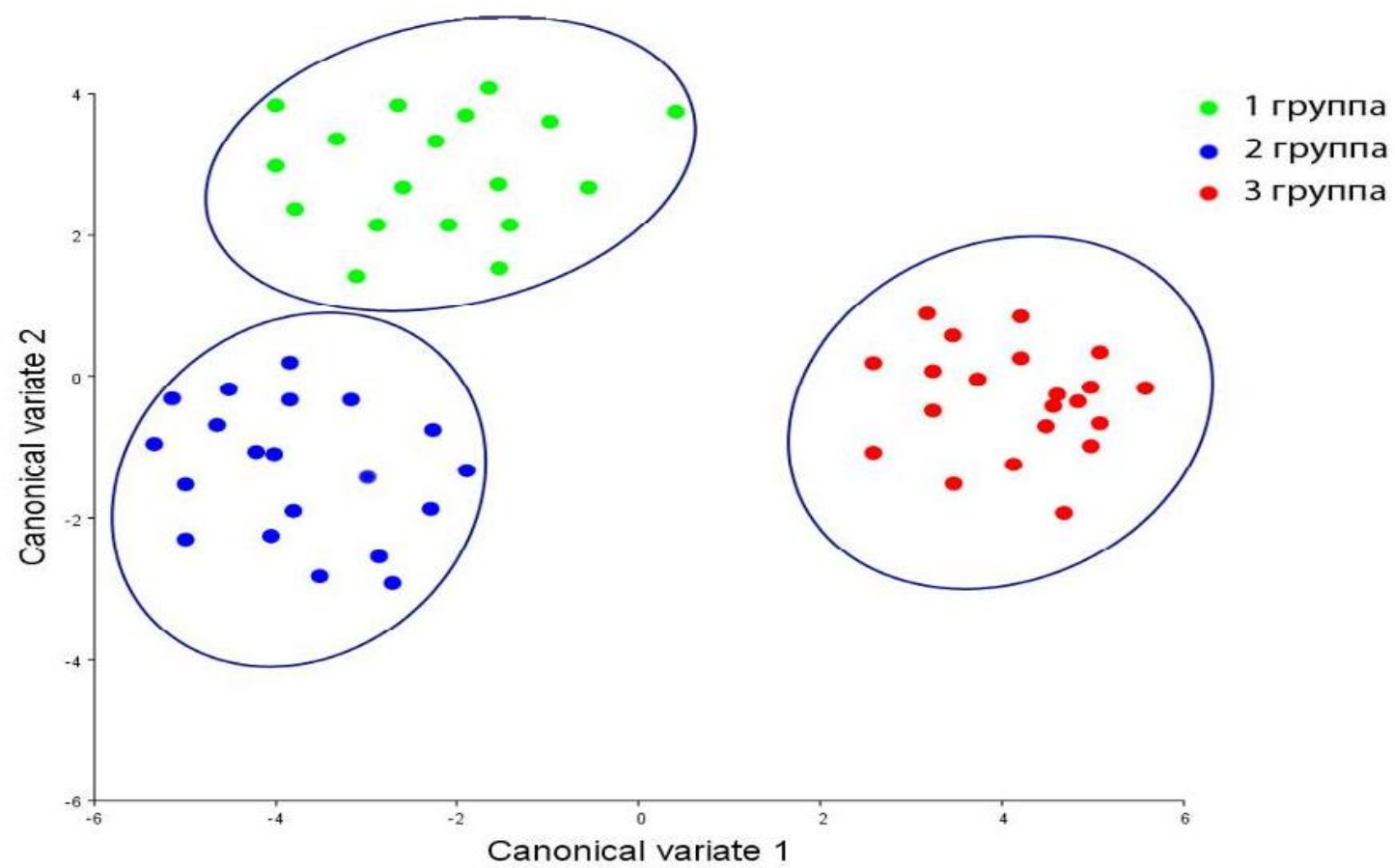

Рис. 3. Размещчение исследуемых групп в плоскости первой (CV1) и второй (CV2) канонических переменных 
Морфофункииональное состояние нижнечелюстных костей лабораторных белых крыс при 1- $u$ 3-месячном воздействии бисфосфонатов в дозах, вызываютих остеонекротические изменения

\section{Таблииа 1}

Результаты канонического анализа составляющей формы нижнечелюстной кости

(* - канонические коэффициенты, имеющие наибольшие абсолютные значения)

\begin{tabular}{|c|c|c|c|c|c|}
\hline & CV1 & CV2 & & CV1 & CV2 \\
\hline $\mathrm{X} 1$ & $74,9364 *$ & $226,6100^{*}$ & $\mathrm{X} 12$ & $-2,9114$ & 28,2889 \\
\hline $\bar{Y} 1$ & $\begin{array}{l}-50,3993 \\
\end{array}$ & $-36,1657$ & Y12 & $-36,2360$ & 4,5736 \\
\hline $\mathrm{X} 2$ & $249,065^{*}$ & $174,8872 *$ & $\mathrm{X} 13$ & $120,8962 *$ & $125,274 *$ \\
\hline $\mathrm{Y} 2$ & 127,3279 & $-98,5928^{*}$ & Y13 & $-59,5565$ & 35,9726 \\
\hline $\mathrm{X} 3$ & 28,5196 & $18,9^{\prime}$ & $\mathrm{X} 14$ & 48,2 & $-13,2$ \\
\hline Y3 & $259,909 *$ & 60,0 & Y14 & $-14,4$ & $-22,3$ \\
\hline $\mathrm{X} 4$ & 60,3746 & $114,4037^{*}$ & $\mathrm{X} 15$ & $-64,3751$ & $-92,019^{*}$ \\
\hline Y4 & $-196,88^{*}$ & \begin{tabular}{|l|}
34,6183 \\
\end{tabular} & Y15 & 41,3350 & 5,9673 \\
\hline $\mathrm{X} 5$ & $-124,85^{*}$ & $-23,5030$ & $\mathrm{X} 16$ & 22,8171 & $-58,5458$ \\
\hline Y5 & $-18,1879$ & $105,0779 *$ & Y16 & $-71,9234 *$ & $-118,13 *$ \\
\hline $\mathrm{X} 6$ & 42,3994 & 9,5 & $\mathrm{X} 17$ & 2,21 & $-33,0698$ \\
\hline Y6 & $-10,6977$ & 37,3235 & Y17 & 45,3402 & $210,095 *$ \\
\hline $\mathrm{X} 7$ & $-15,6936$ & $-36,8116$ & $\mathrm{X} 18$ & $-19,7069$ & $134,032 *$ \\
\hline Y7 & 27,2761 & $-38,1525$ & Y18 & $161,4962 *$ & $77,8501^{*}$ \\
\hline $\mathrm{X} 8$ & 111,0298 & $83,7665^{*}$ & $\mathrm{X} 19$ & $83,9764 *$ & $-45,3391$ \\
\hline Y8 & $-69,7915$ & 32,4367 & Y19 & $115,2569^{*}$ & $203,052 *$ \\
\hline $\mathrm{X} 9$ & $-95,5$ & $124,3178^{*}$ & $\mathrm{X} 20$ & $-55,7722$ & $-92,174 *$ \\
\hline Y9 & $143, \mathrm{C}$ & -70, & Y20 & $-199,1$ & $-184,31 *$ \\
\hline $\mathrm{X} 10$ & 77,8 & 115, & $\mathrm{X} 21$ & 209,9 & $112,721^{*}$ \\
\hline $\mathrm{Y} 10$ & 161,4 & $111,2114^{*}$ & Y21 & $-23,1168$ & $71,4919^{*}$ \\
\hline X11 & $-62,2326$ & $-79,5639 *$ & X22 & $103,2823^{*}$ & $-195,44 *$ \\
\hline Y11 & $-160,68 *$ & $136,1615^{*}$ & Y22 & $171,4251 *$ & $-74,747 *$ \\
\hline
\end{tabular}

Результаты факторного анализа распределения факторных нагрузок переменных величин обеих групп животных, характеризующих размерную составляющую (табл. 2), распредели на 2 фактора. Переменные X, Y1, $\mathrm{X} 2, \mathrm{Y} 2, \mathrm{X} 3, \mathrm{X} 4, \mathrm{X} 6, \mathrm{Y} 11, \mathrm{Y} 12, \mathrm{X} 13, \mathrm{Y} 14, \mathrm{Y} 15$, $\mathrm{X} 16, \mathrm{Y} 16, \mathrm{X} 17, \mathrm{X} 18, \mathrm{Y} 18, \mathrm{X} 19, \mathrm{Y} 19, \mathrm{X} 20, \mathrm{Y} 20$, $\mathrm{X} 21, \mathrm{Y} 21, \mathrm{X} 22, \mathrm{Y} 22$ имеют значимые факторные нагрузки от первого фактора и незначимые - от второго (p>0,7). Переменные X7, X8, X10, $\mathrm{X} 11, \mathrm{X} 14, \mathrm{X} 15$ имеют значимые факторные нагрузки от второго фактора и незначимые - от первого. Анализ факторных нагрузок переменных позволяет интерпретировать первый фактор как изменение общих размеров челюсти, второй - как показатель вариаций в форме, включающий как деформационные нарушения, возникшие под действием вводимых исследуемых веществ, так и фенетические различия внутри линии Вистар. Учитывая пропорциональный прирост всех значимых нагрузок по 1 фактору, для анализа мы использовали переменные $1,21,13$, характеризующие длину резца, длину и ширину челюсти. По второму фактору использовалась значимые переменные 6x, D/A, index (рис. 1).

\section{Результаты факторного анализа (метод главных компонент) исследуемых групп животных после вращения пространства главных факторов (Биквартимакс

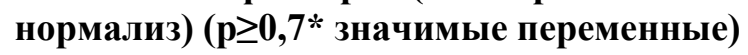

\begin{tabular}{|c|c|c|c|c|c|}
\hline $\begin{array}{c}\text { Переме- } \\
\text { нные }\end{array}$ & Фактор-1 & Фактор-2 & $\begin{array}{l}\text { Переме- } \\
\text { нные }\end{array}$ & Фактор-1 & Фактор-2 \\
\hline $\mathrm{X} 1$ & $0,73775^{*}$ & 0,408214 & X14 & $0,85984 *$ & $-0,11765$ \\
\hline Y1 & 0,63971 & 0,508576 & Y14 & $0,80492 *$ & 0,00221 \\
\hline $\mathrm{X} 2$ & 0,57865 & 0,575798 & 15 & $0,97536^{*}$ & 0,03224 \\
\hline Y2 & $0,81102^{*}$ & 0,254281 & Y15 & $0,94082 *$ & $-0,14876$ \\
\hline X3 & $0,79216^{*}$ & 0,558241 & X16 & $0,96344 *$ & $-0,20541$ \\
\hline Y3 & \begin{tabular}{|l|l|}
0,62899 \\
\end{tabular} & 0,211606 & Y16 & $0,95272^{*}$ & \begin{tabular}{|l|l|} 
& $-0,00887$ \\
\end{tabular} \\
\hline $\mathrm{X} 4$ & $0,84185^{*}$ & 0,478116 & X17 & $0,96271 *$ & $-0,15021$ \\
\hline $\mathrm{X} 5$ & $0,83368^{*}$ & 0,520112 & Y17 & $0,91240^{*}$ & \begin{tabular}{|l|l|} 
& $-0,07704$ \\
\end{tabular} \\
\hline Y5 & 0,54738 & $-0,21336$ & $\mathrm{X} 18$ & $0,97311^{*}$ & $-0,17031$ \\
\hline $\mathrm{X} 6$ & 0,50405 & 0,715365 & Y18 & $0,88991^{*}$ & $-0,11055$ \\
\hline Y6 & 0,27411 & 0,564509 & 19 & $0,9726 * 3$ & \begin{tabular}{|l|l|}
$-0,17079$ \\
\end{tabular} \\
\hline $\mathrm{X} 7$ & 0,26075 & 0,746625 & Y19 & $0,76625^{*}$ & 0,25773 \\
\hline Y8 & $0,80074^{*}$ & \begin{tabular}{|l|}
0,227592 \\
\end{tabular} & $\mathrm{X} 20$ & $0,96134^{*}$ & $-0,11976$ \\
\hline $\mathrm{X} 9$ & 0,64055 & 0,278462 & Y20 & & 0,39027 \\
\hline Y9 & $0,84400^{*}$ & $-0,41972$ & $\mathrm{X} 21$ & $0,95992 *$ & 0,13586 \\
\hline $\mathrm{X} 10$ & 0,17475 & 0,618321 & Y21 & $0,92009^{*}$ & $-0,13149$ \\
\hline Y10 & $0,81987^{*}$ & 0,423774 & $\mathrm{X} 22$ & $0,74915^{*}$ & 0,39161 \\
\hline $\mathrm{X} 11$ & 0,71226 & $-0,13960$ & Y22 & $0,73419 *$ & $-0,45901$ \\
\hline Y11 & $0,94402^{*}$ & 0,193330 & индекс & \begin{tabular}{|l|}
$-0,06996$ \\
\end{tabular} & $0,79069^{*}$ \\
\hline $\mathrm{X} 12$ & $0,76116^{*}$ & 0,131704 & C/A & \begin{tabular}{|l|l|}
0,47094 \\
\end{tabular} & \begin{tabular}{|l|l}
$-0,40989$ \\
\end{tabular} \\
\hline Y12 & $0,97040^{*}$ & 0,092906 & $\mathrm{C} / \mathrm{B}$ & 0,56535 & $-0,53943$ \\
\hline X13 & $0,9054 * 1$ & $-0,38828$ & $\mathrm{D} / \mathrm{A}$ & $-0,38773$ & $0,72526^{*}$ \\
\hline Y13 & $0,93119^{*}$ & 0,291061 & $\mathrm{D} / \mathrm{B}$ & $-0,26990$ & $0,88953^{*}$ \\
\hline $\begin{array}{c}\text { Общая } \\
\text { диспер- } \\
\text { сия }\end{array}$ & \multicolumn{2}{|c|}{27,36606} & $\begin{array}{c}\text { Общая } \\
\text { диспер- } \\
\text { сия }\end{array}$ & \multicolumn{2}{|c|}{7,097452} \\
\hline $\begin{array}{c}\text { Доля } \\
\text { общей } \\
\text { диспер- } \\
\text { сии }\end{array}$ & \multicolumn{2}{|c|}{0,59491} & $\begin{array}{c}\text { Доля } \\
\text { общей } \\
\text { диспер- } \\
\text { сии }\end{array}$ & \multicolumn{2}{|c|}{0,154292} \\
\hline
\end{tabular}

Результаты MANOVA показали, что контролируемые факторы и их взамодействие имеют значимые различия $\mathrm{F}_{\text {возр }}=422,5\left(\mathrm{p}<10^{-3)}\right)$, $\mathrm{F}_{\text {преп }}=321,4\left(\mathrm{p}<10^{-3)}\right)$ и $\mathrm{F}_{\text {возр-преп }}=120,8\left(\mathrm{p}<10^{-3)}\right)$, что позволило нам продолжить дальнейшее статистическое исследование с помощью факторной и однофакторной ANOVA по каждой зависимой переменной и выбрав фактор $\mathrm{F}_{\text {возр-преп }}$ (группа-серия), провести множественные сравнения средних, используя апостериорный критерий Ньюмана-Кейлса.

Анализируя значимые результаты выделенных при помощи факторного анализа показателей размерной составляющей третьей группы животных (получавших в течение месяца памидроновую кислоту) с показателями 1-й контрольной группы, отмечаем увеличение 
размерной составляющей по всем исследуемым параметрам длины резца на $17,7 \%\left(\mathrm{p}_{1-3}<10^{-3}\right)$, длины челюсти - на $12,5 \%,\left(\mathrm{p}_{1-3}<10^{-3}\right)$ и ширины челюсти на $13,4 \%\left(\mathrm{p}_{1-3}<10^{-3}\right)$. Индекс прочности уменьшился на $15,1 \%\left(\mathrm{p}_{1-3}<10^{-3}\right)$. Положение промера 6x, характеризующего положение угла челюсти, также увеличилось в вентральном направлении на 9,2\% $\left(\mathrm{p}_{1-3}<10^{-3}\right)$ (рис. 4, 5). Другая картина наблюдается при анализе остеометрических показателей нижней челюсти животных 4 серии (получавших в течении месяца памидроновую кислоту по сравнению с аналогичными показателями 1 группы 2 серии серии: длина резца была меньше на 9,9\% $\left(\mathrm{p}_{2-4}<10^{-3}\right)$, длина и ширина челюсти $-8,9 \% 8,7$ $\left.\left(\mathrm{p}_{2-4}<10^{-3}\right)\right)$. Вершины угла челюсти также на $9,2 \%\left(\mathrm{p}_{2-4}<10^{-3}\right)$ располагались дорзальнее аналогичного показателя животных второй серии первой группы. Также отмечается уменьшение индекса прочности на 14,01\% $\left(\mathrm{p}_{2-4}<10^{-3}\right)$, (рис 4,5). Существенных значимых различий между резцово-масетериальным индексом по сравнению с аналогичным пока- зателям животных первой группы 2-й серии, не наблюдалось $\left(\mathrm{p}_{1-3}=0,575^{\circ}\right),\left(\mathrm{p}_{2-4}=0,585^{\circ}\right)$, (рис. 5). При сравнении остеометрических показателей животных 5 группы (подвергнутых воздействию модельной примеси в течение 1 месяца), отмечаем увеличение всех параметров, характеризующих линейную размерность нижнечелюстной кости длины резца на $14,1 \%$ $\left(\mathrm{p}_{1-5}<0,10^{-3}\right)$, длины челюсти - на 9,6\% $\left(\mathrm{p}_{1-5}<0,10^{-3}\right)$, и ширины челюсти - на $6,2 \%$ $\left(\mathrm{p}_{1-5}<0,10^{-3}\right)$ (рис. 4, 5). В 6-й серии отмечаем низкие значения исследуемых линейных показателей. Длина резца челюсти - на 11,6 \% $\left(\mathrm{p}_{1-6}<0,10^{-3}\right)$, длина челюсти - на $13,1 \%$ $\left(\mathrm{p}_{1-6}<0,10^{-3}\right)$, и ширина - на $14,1 \%\left(\mathrm{p}_{1-6}<0,10^{-3}\right)$ меньше аналогичных показателей 2-й серии (рис. 4). Индекс прочности у животных исследуемых групп уменьшился на $25 \%$ $\left(\mathrm{p}_{1-5}<0,10^{-3}\right) \quad$ и $30 \%\left(\mathrm{p}_{1-6}<0,10^{-3}\right)$. Массетериально-резцовый индекс в обеих группах уменьшился на $14,2\left(\mathrm{p}_{1-5}<0,10^{-3}\right)$ и $6 \%$ $\left(\mathrm{p}_{1-6}<0,10^{-3}\right)$ по сравнению с аналогичными показателями контрольной грvппы (рис. 4. 5).
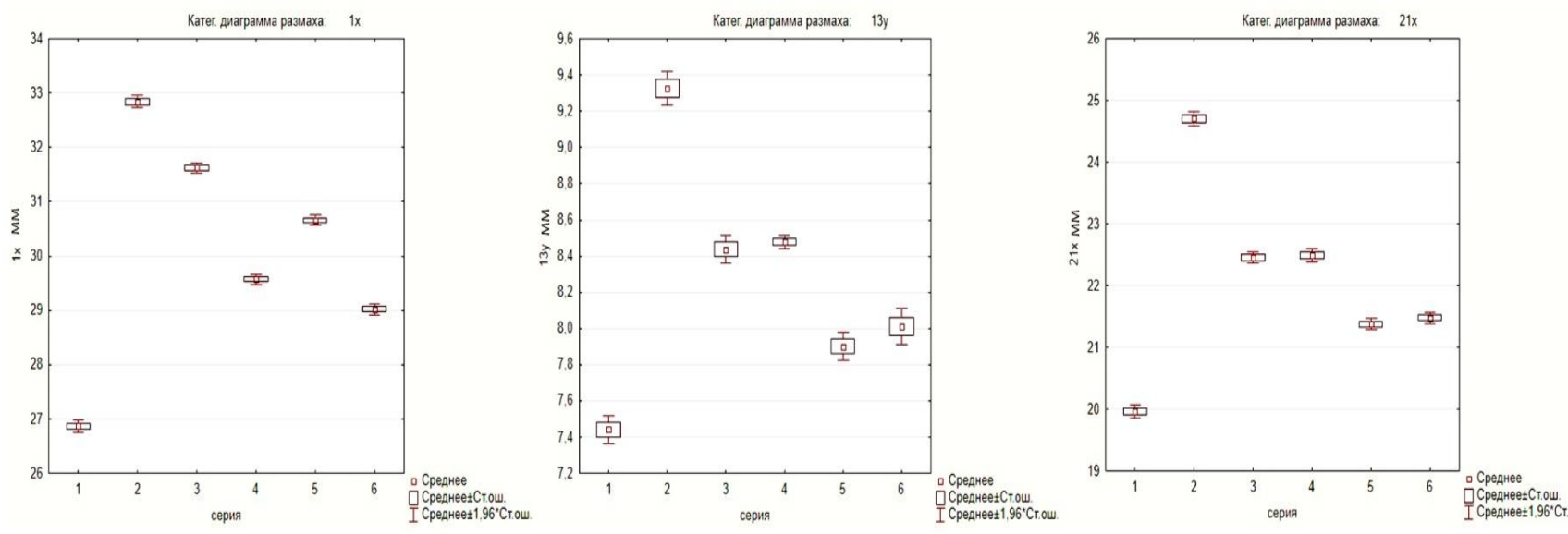

Рис 4. Категоризованные диаграммы размаха промеров $1 x, 21 x$, и $13 y$ исследуемых групп животных
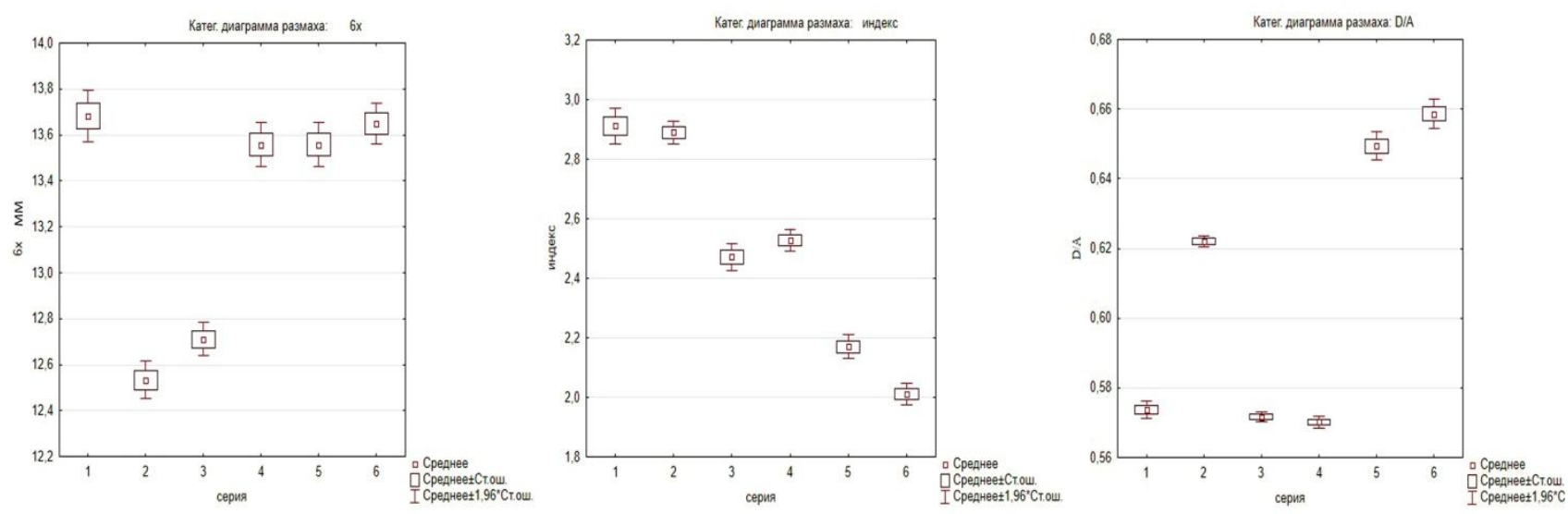

Рис 5. Категоризованные диаграммы размаха индекса прочности (IR), резиово-масетериального индекса (AD) и промера 6х исследуемых групп животных 
Морфофункииональное состояние нижнечелюстных костей лабораторных белых крыс при 1- $и$ 3-месячном воздействии бисфосфонатов в дозах, вызываютих остеонекротические изменения

\section{Обсуждение результатов}

В результате проведенного исследования эффектов воздействия исследуемых остеотропных соединений фосфора наблюдались следующие изменения в формообразовании и росте нижнечелюстных костей исследуемых животных.

В течение месячного срока использования исследуемых веществ выраженные остеостимулирующие свойства обоих исследуемых веществ, преобладающие в 3-й группе животных, находившихся под воздействием памидроновой кислоты. Это подтверждается преобладанием исследуемых показателей, характеризующих размерную составляющую нижней челюсти: длины резца - на $3 \%$ $\left(\mathrm{p}_{3-5}<10^{-3}\right)$, длины и ширины челюсти - на $5,4 \%$ $\left(\mathrm{p}_{3-5}<10^{-3}\right)$ и $6,8 \%\left(\mathrm{p}_{3-5}<10^{-3}\right)$ в сравнении с показателями пятой группы (рис. 4, 5), что соответствует данным литературы и нашим ранее опуб-ликованным результатам [5, 14]. Высокие значения, характеризующие размер резцов нижнечелюстной кости в обеих экспериментальных группах, связаны со снижением активности животных (рис. 4, 5). Это вызвано «реакцией острой фазы», присущей в первые недели воздействия всем амино- и бифосфонатным соединениям, на фоне которой снижается активность животных, уменьшается потребляемая пища и снижается интенсивность стачивания резцов [14]. Следует отметить уменьшение индекса прочности на $12 \%$ $\left(\mathrm{p}_{3-5}<10^{-3}\right)$ по сравнению $\mathrm{c}$ аналогичными показателями животных 3-й экспериментальной группы, что свидетельствует о более интенсивном накоплении минерального компонента (рис. 4, 5).

В трехмесячный период воздействия отмечается торможение процессов роста и искажение процессов формообразования. Торможение ростовых процессов, прослеживаемое в обеих опытных группах, наиболее выражено у животных, получавших модельную примесь по показателям, характеризующим размерную составляющую. При этом длина и ширина челюсти на $5 \%\left(\mathrm{p}_{4-6}<10^{-3}\right)$, а на $6,8 \%$ $\left(\mathrm{p}_{4-6}<10^{-3}\right)$ были ниже аналогичных показателей 4-й экспериментальной группы (рис. 4, 5). Искажение процессов формообразования происходит в виде утолщения резцового и молярного отделов и сглаженности части рельефа нижнечелюстной кости в обеих исследуемых экспериментальных группах. Это проявляется в «выравнивании» кривизны в отделах margo interalveolaris, incisura angylaris, angulus mandibulae, incisura mandibulae и по всей поверхности нижнечелюстной кости, у животных 3- и 4-й опытных групп и подтверждается результатами канонического анализа в виде высоких значений канонических коэффициентов по первой канонической переменной X6, Y6, X9, Y9, X13, Y13 и низким показателем морфофункционального массетерно-резцового $-M I=D / A(M M)$ индекса, который на $13,6 \%\left(\mathrm{p}_{4-6}<10^{-3}\right)$ превышает аналогичный показатель 6-й серии животных (табл. 1,3, рис. 4, 5). Помимо сглаженности рельефа у животных 5- и 6-й экспериментальных групп отмечается деформация резцово-молярного отделов, в результате чего глубина angulus mandibulae остаётся неизменной на протяжении всего исследования, а положение его вершины смещается в вентральном направлении, что подтверждается отсутствием статистически значимых различий промеров 6x с аналогичными показателями животных второй группы $\left(\mathrm{p}_{3-5}=0,269\right)$, $\left(\mathrm{p}_{4-6}=0,07\right) \quad$ (рис. 4, 5). Подобные изменения нижнечелюстных костей и отставание в росте подтверждаемое более низкими значениями индекса прочности (величина которого на $20 \%$ $\left(\mathrm{p}_{4-6}<10^{-3}\right)$ меньше аналогичного показателя животных четвёртой группы), результатами канонического анализа в виде более высоких значений канонических коэффициентов по первой и второй каноническим переменным $\mathrm{X} 1, \mathrm{X} 2, \mathrm{Y} 2, \mathrm{X} 4, \mathrm{Y} 5, \mathrm{X} 8, \mathrm{X} 9, \mathrm{X} 10, \mathrm{X} 11, \mathrm{Y} 11$, $\mathrm{X} 13, \mathrm{X} 15, \mathrm{X} 16, \mathrm{Y} 19, \mathrm{X} 16, \mathrm{Y} 17, \mathrm{X} 18, \mathrm{Y} 18, \mathrm{Y} 19$, $\mathrm{X} 20, \mathrm{Y} 20, \mathrm{X} 21, \mathrm{Y} 21, \mathrm{X} 22, \mathrm{Y} 22$ (табл. 1, рис. 4, 5), видимо, связаны с более высокой фармакологической активностью модельной примеси, вызывающей более интенсивное отложение минеральной составляющей с преимущественным накоплением в зонах роста и активной функциональной перестройки нижней челюсти, а возможно, и обладающей общетоксическими эффектами, опосредованно влияющими на остеогенные процессы $[4,5,14]$. Следует отметить уменьшение длины резцов, что свидетельствует о восстановлении функциональной активности животных.

Таким образом, в ранние сроки воздействия, исследуемые бисфосфонатные соединения обладают выраженным остеостимулирующим действием, несмотря на наличие «реакции острой фазы», возникающей в первые две недели использования [14]. В поздние сроки исследования вещества вызывают торможение роста и искажение процессов формообразования, что обусловлено фармакологическими свойствами исследуемых веществ, блоки- 
рованием костной резорбции и широким спектром возможных токсических реакций со стороны мочевыделительной, пищеварительной, и сердечно-сосудистой систем [14].

\section{Выводы}

В месячный период исследования препараты оказывают разноплановые эффекты, где процессы токсического действия и интенсивной минерализации накладываются на интенсификацию ростовых потенций, наиболее выраженные при применении памидроновой кислоты.

У животных, получавших памидроновую кислоту в течение трёх месяцев, отмечается торможение процессов роста на фоне искажения процессов формообразования, что проявляется сглаженностью рельефа и утолщением тела нижней челюсти в области резцово-молярного отделов.

У животных, подвергнутых воздействию модельной примесью, на фоне низких ростовых потенций также отмечается сглаженность рельефа нижнечелюстных костей и деформация формы в виде недоразвития молярнорезцового сегмента с преобладанием последнего.

\section{Литература}

1. Rustemeye, J., Melenberg, A., Junker, K., Sari-Rieger, A. Osteonecrosis of the maxilla related to long-standing methamphetamine abuse: a possible new aspect in the etiology of osteonecrosis of the jaw. Oral and Maxillofacial Surgery; 2014, 18 (2): c 237-41. http://doi.org/10.1007/s10006-014-0449-2

2. Favus, M. D. Bisphosphonates for osteoporosis. N. Engl. J. Med, 2010; 363: c 2027-35.

3. Заславская, Н. А., Дробышев, А. Ю., Волков, А. Г. Опыт лечения остеонекрозов челюстей у пациентов, получающих антирезорбтивную терапию (бисфосфонаты, «Деносумаб»). Cathedra, 2014; (47): c 32-34.

4. Semen, O. Mostovoy, Viktor, F. Shul'gin, Elena, M. Maksimova, Igor, A. Nauhatsky et al. Mineralizing process and morphological structure of the femoral bone in rats under influence of aminophosphonates. $J$ Exp Integr Med, 2014; 4(2): c 81-84.

5. Мостовой, С. О., Шульгин, В. Ф., Пешков., М., .В. Патоморфологическое исследование побочных эффектов воздействия аминобисфосфонатов на нижнечелюстные кости лабораторных белых крыс. Клиниеская $u$ экспериментальнаяморфология; 2017, № 1, с 41-46.

6. Врублевський, А. Г. Наркомании $и$ токсикомании: клинические формы наркоманий токсикоманий Лекции по наркологии. [Ред. Н. Н. Иванц]; Медицина: Москва; 2000; с 241.

7. Рыболовлев, Ю. Р., Рыболовлев, Р. С. Дозирование веществ для млекопитающих по константе биологической активности. Доклады $A H$ CCCP, 1979; 247 (6): c 1513-1516.

8. European convention for the protection of vertebrate animals used for experimental and other scientific purpose: Council of Europe 18.03.1986. Strasbourg, 1986. p 52.

9. Rohlf, F. J. tpsDig. N. Y.: State Univ. at Stony Brook. Version 1.23. 2001b. [Electronic resource]. Mode of access: http://life.bio.sunysb.edu/morph.

10. Кендалл, М., Стьюарт, А. Многомерный статистический анализ и временные ряды. Москва, 1976, с 400-437.

11. Anderson, P. S. L., Renaud, S., Rayfield, E. J. Adaptive plasticity in the mouse mandible. $B M C$ Evolutionary Biology, 2014, V. 14, p 85-93.

12. Hiiemae, K. M. The structure and function of the jaw muscles in the rat (Rattus norvegicus L.) III. The mechanics of the muscles. Zoological Journal of Linnean Society, 1971, V. 50, p 111-132.

13. Riesenfeld, A. Metatarsal robusticity in biuedal rats. Am'. J. Phys. Anthropol., 1972, 36(2), p 229-233.

14. Craig, F., Mansoor, H., Janet, H. Acute phase response and mineral status following low dose intravenous zoledronic acid in children. Bone, 2007, No 41, p 366-370. 